\title{
Can Elastosonography Be Useful in Improving Diagnosis and Prognosis of Acute Muscle Injuries?
}

\author{
Marco Cianforlini ${ }^{1}$ Serena Ulisse ${ }^{2}$ Valentino Coppa ${ }^{1} \quad$ Marco Grassi ${ }^{1}$ Marco Rotini ${ }^{1} \quad$ Antonio Gigante $^{1}$ \\ ${ }^{1}$ Department of Clinical and Molecular Sciences, School of Medicine, \\ Università Politecnica delle Marche, Ancona, Italy \\ 2 Department of Radiological Sciences, Università Politecnica delle \\ Marche, Ancona, Italy \\ Address for correspondence Marco Cianforlini, MD, Department of \\ Clinical and Molecular Sciences, Università Politecnica delle Marche, Via \\ Tronto 10/60126, Ancona, Italy (e-mail: marco.cianforlini@libero.it).
}

Joints 2018;6:116-121.

\begin{abstract}
Keywords

- football

- muscle injuries

- muscle pain

- ultrasound

- elastosonography

Purpose The objective of this study was to investigate the ability of elastosonography (USE) in the identification of different grades of muscular injuries, comparing its effectiveness with traditional ultrasound (US) survey and by relating the results to the clinical classification of muscular pain.

Methods In the period between August 2014 and May 2016, we conducted a prospective cohort study on a population of 34 young male professional athletes belonging to the same under-17 football club (Ancona 1905). Injuries were recorded according to location, type, mechanism, recurrence, and whether they occurred with or without contact. Muscle pain was classified, after a physical examination, according to the classification of Mueller-Wohlfahrt et al. All athletes were evaluated by musculoskeletal US and USE in hours following the trauma/onset of pain.

Results Seventy injuries were documented among 19 players. Muscle/tendon injuries were the most common type of injury (49\%). USE showed areas of edema in nine lesions that were negative at the US examination and previously classified as fatigue-induced muscle disorders. These nine players took more time to return to physical activity compared with others with injuries classified into the same group, but negative at USE evaluation. Conclusion USE is a valuable aid in the diagnosis and prognostic evaluation of muscle injury, as it detects pathologic changes that are not visible with the B-mode US. Level of Evidence This is a Level III, observational cohort study.
\end{abstract}

\section{Introduction}

Muscular injuries are very frequent in sports, especially in football. ${ }^{1-4}$ The diagnosis of muscular injuries is clinical and mostly based on history of injury mechanism and symptoms and on physical examination. An accurate diagnosis supported by objective instrumental data is crucial to select the proper treatment, to prevent future damage and to allow an early return to play in accordance with the biological healing time.

received

September 5, 2017

accepted

May 7, 2018

published online

June 22, 2018
DOI https://doi.org/

$10.1055 / \mathrm{s}-0038-1660814$. ISSN 2282-4324.
Despite considered complementary studies, musculoskeletal ultrasound (US), elastosonography (USE), and magnetic resonance imaging (MRI) can assess diagnosis and prognosis more significantly than the clinical examination. ${ }^{5-7}$ In 2012, Mueller-Wohlfahrt et $\mathrm{al}^{8}$ published a new classification focusing on muscle pain, related or not to the presence of a lesion, using clinical examination, US and MRI.

The USE allows excellent follow-up assessment of muscle and tendon injuries by demonstrating the recovery of

Copyright $\odot 2018$ Georg Thieme Verlag License terms KG Stuttgart · New York
(1) $\ominus \circledast$ 
elasticity in the injured areas. However, no previous studies investigated the diagnostic value of USE in acute muscle and tendon injuries.

The objective of this study was to investigate the ability of USE to discriminate the different grades of muscular injuries by comparison with standard US and by correlation with the clinical classification of muscular pain. Our hypothesis was that USE could provide additional information and therefore allow for a better assessment of muscle injuries than the standard US.

\section{Methods}

In the period between August 2014 and May 2016 (two football seasons), we conducted a nonrandomized prospective cohort study on a population of young male professional athletes belonging to the under-17 football club (Ancona 1905). The team has been monitored by the same medical team belonging to the Orthopaedic Clinic of Ospedali Riuniti, Ancona. Only players who did not have muscle injuries before the beginning of the research were included in the study. If a player had joined or left the team during the season, the resulting data were adjusted considering the number of days he was observed. The study was approved by the loca Institutional Review Board and all the athletes signed an informed consent to enter the study.

Injury recording followed the guidelines for injury definitions and data collection procedures in studies on soccer injuries provided by Fédération Internationale de Football Association (FIFA)-Medical Assessment and Research Centre (F-MARC). The injury criteria adopted in this study followed the definition of time loss provided by the Medical Committee of Union of European Football Associations: "an injury that occurred during a scheduled training session or match that caused absence from the next training session or match." Injuries were recorded by the rehabilitative team of the club, who was trained to use the F-MARC form at the beginning of the season. The injury event was recorded immediately after it occurred, and the team's physiotherapists recorded the match and training hours. Injuries were then classified either as "requiring medical attention," as "time loss" when causing the player to miss training or competition, as "early recurrence" if occurring within 2 months from the return to play and as "late recurrence" if occurring from 2 to 12 months from the return to play.

Injuries were described as frequency and percentage according to location, type, mechanism, recurrence, and whether they occurred with or without contact. The incidence of injury during matches and training was reported as the number of injuries per 1,000 hours played. Injury recording considered the moment at which the injury occurred (match or training), as well as severity, location, type, mechanism, and recurrence.

Injury severity was classified according to the number of days lost by the player between the day of the injury and the return to full participation in team training, and the availability to be selected to play; it was classified as follows: minimal (15 days), mild (6-10 days), moderate (11-28 days), and severe ( $>28$ days). ${ }^{8,9}$ The number and severity of injuries were calculated as ratio with the number of days that each player was monitored. Since a player could enter or leave the team during the study, this normalization procedure was chosen to consider differences of monitoring time of the players in the quantity and severity (lost days) of injuries.

Location of injury was defined according to the following categories: head/neck, upper limbs, trunk, and lower limbs.

The type of injury was classified as fracture/bone stress, joint (nonbone)/ligament, muscle/tendon, contusions, laceration/skin injury, and others. ${ }^{8}$

The mechanism of injury was classified as traumatic (i.e., resulting from a specific and identifiable event) or overuse (i.e., caused by repeated microtraumas, even without a simple and identifiable event).

As for muscle injuries, muscle pain was classified, after a physical examination, according to the classification of Mueller-Wohlfahrt et $\mathrm{al}^{8}$ ( - Table 1). Physical examination evaluated the location of spontaneous pain, tenderness to palpation of the concerned muscle, the presence of muscle hypertonicity,

Table 1 Classification of muscle disorders according to Mueller-Wohlfahrt et al ${ }^{8}$

\begin{tabular}{|l|l|l|l|}
\hline $\begin{array}{l}\text { A. Indirect muscle } \\
\text { disorder/injury }\end{array}$ & $\begin{array}{l}\text { Functional muscle } \\
\text { disorder }\end{array}$ & $\begin{array}{l}\text { Type 1: Overexertion-related muscle } \\
\text { disorder }\end{array}$ & $\begin{array}{l}\text { Type 1A: Fatigue-induced muscle } \\
\text { disorder } \\
\text { Type 1B: Delayed-onset muscle } \\
\text { soreness (DOMS) }\end{array}$ \\
\cline { 3 - 4 } & $\begin{array}{l}\text { Type 2: Neuromuscular muscle } \\
\text { disorder }\end{array}$ & $\begin{array}{l}\text { Type 2A: Spine-related } \\
\text { neuromuscular Muscle disorder } \\
\text { Type 2B: Muscle-related } \\
\text { neuromuscular Muscle disorder }\end{array}$ \\
\cline { 2 - 4 } & $\begin{array}{l}\text { Structural muscle } \\
\text { injury }\end{array}$ & Type 3: Partial muscle tear & $\begin{array}{l}\text { Type 3A: Minor partial muscle tear } \\
\text { Type 3B: Moderate partial muscle } \\
\text { tear }\end{array}$ \\
\cline { 2 - 4 } & $\begin{array}{lll}\text { Subtotal or complete muscle tear } \\
\text { Tendinous avulsion }\end{array}$ \\
\cline { 2 - 4 } & Type 4: (Sub)total tear & \\
\hline
\end{tabular}


functional impairment, decreased muscle strength, increased harmed muscle volume, subcutaneous swelling, and the presence of subcutaneous ecchymosis, if any.

All athletes were evaluated in the hours following the trauma/onset of pain (from 6 to a maximum of 72 hours) using a diagnostic US system (Philips iU22; Philips Healthcare, Bothell, Washington, United States) and classified according to Mueller-Wohlfahrt et al if one or more clinical or instrumental parameters experienced any change, by differentiating functional disorders from structural injuries.

Musculoskeletal US and USE examination were performed by two radiologists experienced in musculoskeletal US. The USE was performed with free-hand technique giving to the US transducer rhythmic manual compressions on tissues under examination. A rectangular region of interest (ROI) was used to represent the entire structure into consideration. For the feedback, the images obtained from at least three compression/relaxation cycles were recorded, using the intermediate images in the compression phase of each cycle since the initial and final images are inaccurate.

The athletes were evaluated again clinically and by musculoskeletal US and USE after 7, 15, and 30 days from the date of trauma/onset of pain. Anechogenic areas of edema or USE red areas, indicating higher tissue elasticity related to the edema or hematoma, were highlighted.

A transducer was used to obtain specific information in USE imaging. By exerting low pressure with the transducer in the ROI, it was possible to determine a proportional correlation between pressure and deformation. The size of the ROI determined by the examiner should exceed $5 \mathrm{~mm}$ all around the explored lesion (-Fig. 1).

\section{Results}

Thirty-seven male athletes were initially evaluated. Two athletes were excluded due to a previous injury. During the season, three new athletes joined the team, and no one suffered musculoskeletal injuries. In addition, four athletes left the team during the season and consequently were not followed up for the entire period. At the end of data collection, 34 athletes were examined, with an average age of 17.2 years ( $\mathbf{-}$ Table $\mathbf{2}$ ).

Overall, 318 team-training sessions (683.7 training hours) and 56 matches (70.2 match hours) were recorded during the
Table 2 Baseline characteristics of study population

\begin{tabular}{|l|l|}
\hline Height $(\mathrm{cm})$ & $178.57 \pm 5.65$ \\
\hline Weight $(\mathrm{kg})$ & $64.36 \pm 6.48$ \\
\hline Body mass index $\left(\mathrm{kg} / \mathrm{m}^{2}\right)$ & $20.20 \pm 3.82$ \\
\hline No. of matches & $56(26+30)$ \\
\hline No. of training sessions & $318(150+168)$ \\
\hline Match hours & $70.2(31.2+39)$ \\
\hline Training hours & $683.7(330+369.6)$ \\
\hline
\end{tabular}

study period. Seventy injuries were documented in 19 players (54.3\%). All injuries were "medical attention injuries." Injury incidences were 38.7 and 12.8 for match and training injuries, respectively. Forty-eight injuries (71.6\%) led to "time loss," and incidences for match and training were 27.3 and 8.6 per 1,000 hours, respectively.

Traumatic injuries represented $38.6 \%$ of all injuries and the remaining were noncontact injuries due to overuse, fatigue induced, or other clinical causes. The majority of injuries (73\%) affected lower extremities. Muscle/tendon injuries were the most common type of injury (49\%), followed by fracture/bone/stress (17\%) (-Fig. 2). Furthermore, there were three muscle reinjuries $(8.8 \%)$ due to overuse; of these, two were early and one was late recurrence. The most frequent locations of muscle injuries were hamstrings in 11 cases (32\%), triceps in 9 (26\%), quadriceps in 7 (21\%), anterior and lateral calf in $4(12 \%)$, and adductors in $3(9 \%)$.

All muscle injuries were time loss injuries, of which $41 \%$ (14 athletes) resulted in minimal absence (5 days or less). The remaining 20 injuries (59\%) resulted in absence of more than 6 days. Of these, 12 (35\%) were classified as mild (610 days), 4 (12\%) as moderate (11-28 days), and $4(12 \%)$ as severe ( $>28$ days).

At the first clinical assessment, 23 muscle disorders were classified as functional pain, while the remaining 11 were classified as structural pain (8 due to indirect trauma and 3 due to a direct trauma).

On subsequent evaluation (48 hours from injury), on the basis of clinical and US examination, 18 muscle pain were classified as fatigue-induced muscle disorder (FIMD, 1a), 4 as delayed-onset muscle soreness (1b), 1 as spine-related
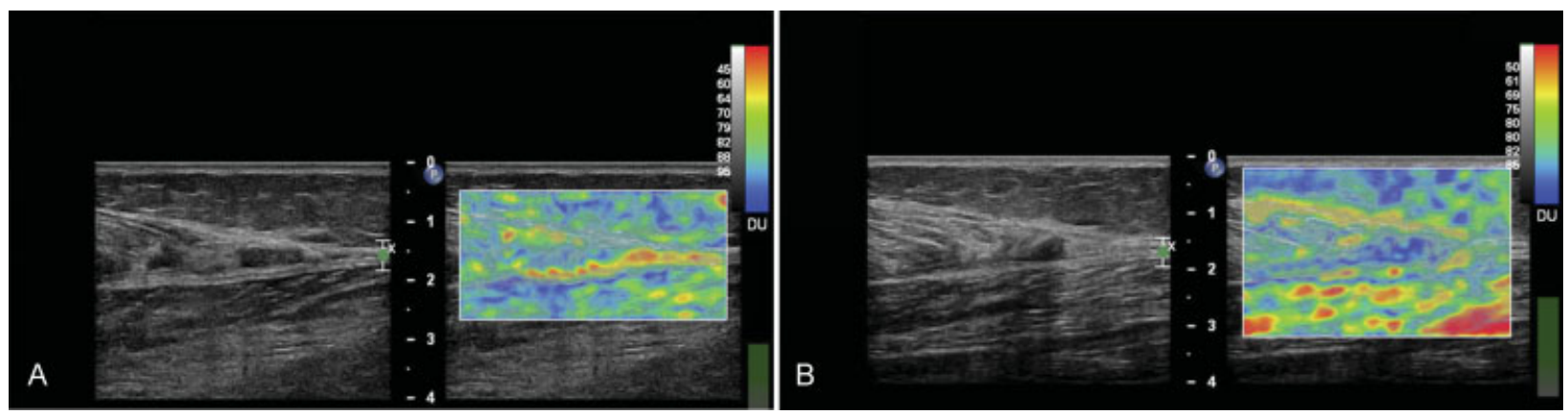

Fig. $13 \mathrm{~b}$ injury of the medial head of the gastrocnemius in a professional 17-year-old football player. US and USE performed at 48 hours (A) and 15 days (B) after trauma. US, ultrasound; USE, elastosonography. 


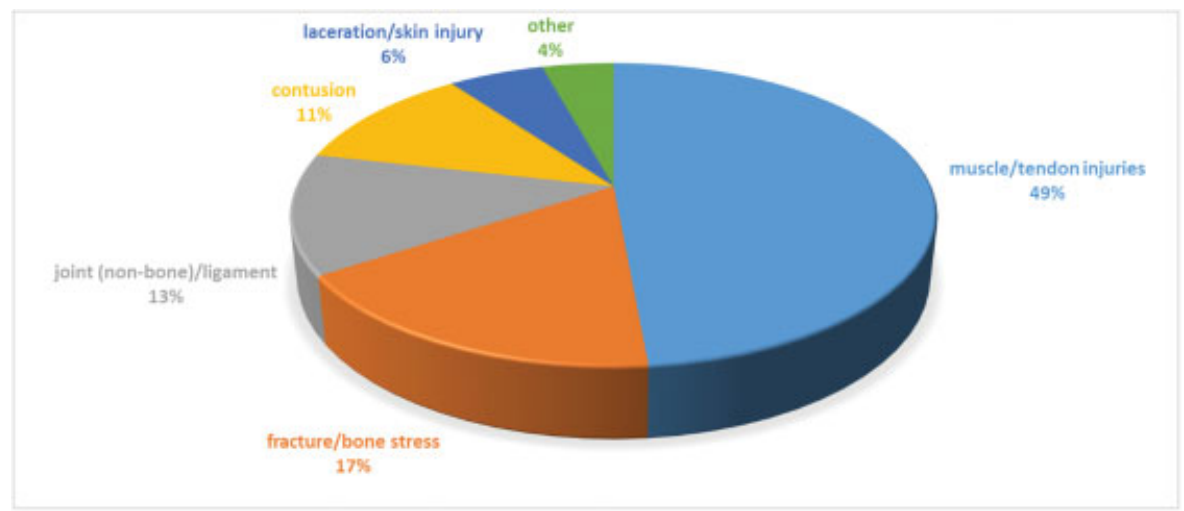

Fig. 2 Classification of injuries according to the type of injury.

neuromuscular disorder (2a), 2 as structural lesions (3a), 6 as $3 \mathrm{~b}$, and 3 as injuries from direct trauma ( - Fig. 3). The USE evaluations confirmed the clinical diagnosis and US findings. However, areas of edema (red areas, indicating increased tissue elasticity related to the edema) were identified in nine patients, who had been classified as FIMD (1a) (-Fig. 4).

\section{Discussion}

Muscle damage is defined as a lesion of muscle fibers without involvement of the extracellular matrix, blood perfusion, and innervation. The amount of muscle tissue affected, the extent, and location of the effusion define the severity of muscle trauma. ${ }^{10-14}$

Clinically, discerning 1a lesions from 3a lesions proves challenging, especially in the early phase when blood extravasation could possibly be unnoticeable. In this case, diagnosis should rely not only on the clinical features of the lesion but also on US survey results, approximately 48 to 72 hours from the trauma.
Musculoskeletal US and USE are able to provide clinicians with adequate data about muscle injury diagnosis. B-mode US does not play a large role in assessing elongations and contractures because the absence of fiber lesions entails that there forms no hematoma but only a moderate and diffuse intramuscular edema. USE measures tissue deformation as a response to the application of an external force, the assumption being that the deformation will be lower in rigid tissues, compared with elastic, soft tissues.

Elastosonography is currently available in all US scanners. It is based on the principle that a distinctive vibration is associated with the degree of elasticity of the tissue being examined. The vibration, obtained by moderate tissue compression with the probe, is translated by the software into a color map. Elastosonography allows excellent follow-up assessment of muscle and tendon lesions by demonstrating the recovery of elasticity in areas that have suffered injury. ${ }^{15}$ Therefore, edema is not clearly depicted by US. On the contrary, USE could show loss of elasticity of muscle component during compression, even in acute phase of an indirect muscular trauma.

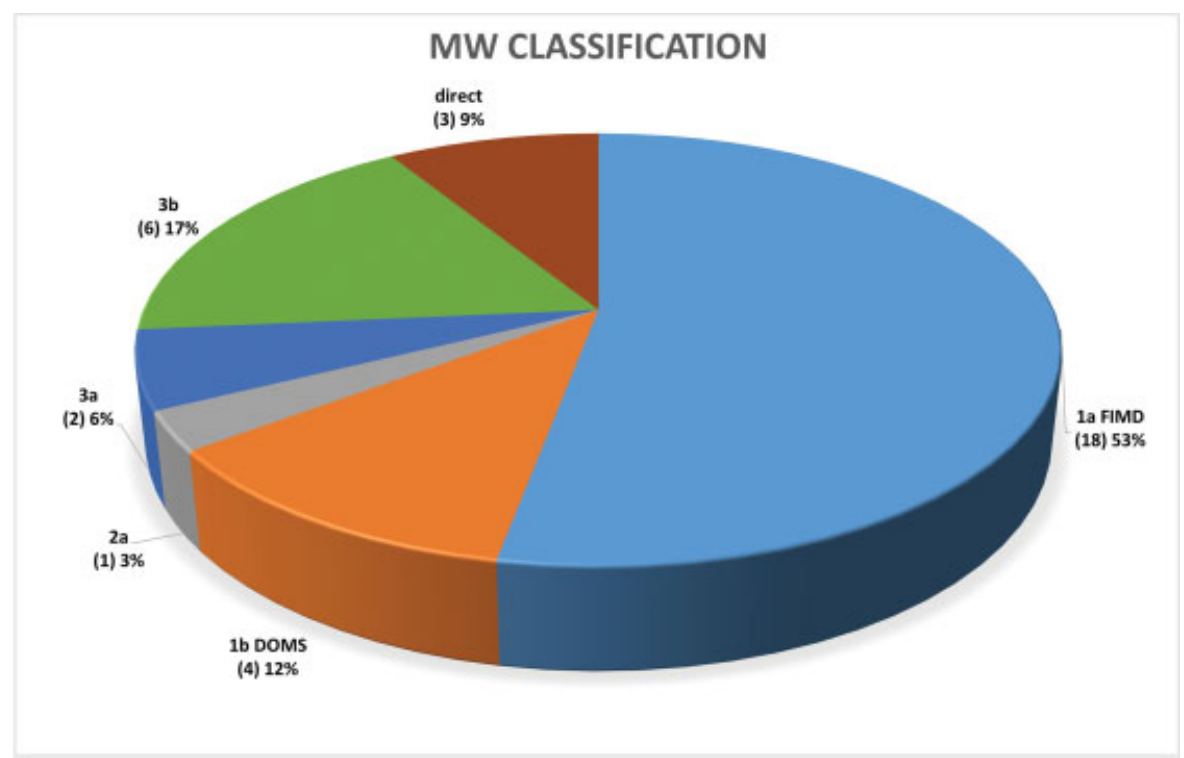

Fig. 3 Classification of muscle injuries according to Mueller-Wohlfahrt et al. ${ }^{8}$ FIMD, fatigue-induced muscle disorder; MW, Mueller-Wohlfahrt. 


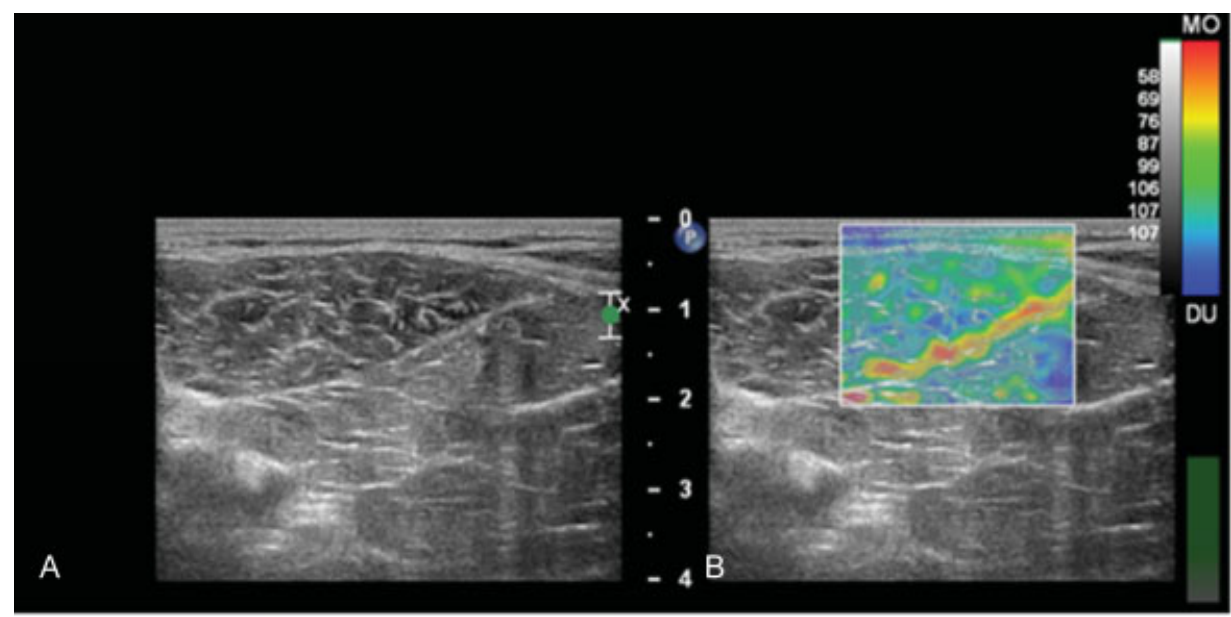

Fig. 4 Longitudinal scanning of vastus medialis muscle. Indirect trauma in a 17-year-old football player. (A) B-mode US shows no signs of edema or muscle injury. (B) USE shows red area (box) of soft tissue localized in the same area of the pain referred by the athlete. US, ultrasound; USE, elastosonography.

An early postinjury US 36 to 48 hours after the muscle trauma can provide helpful information about any existing muscle structure problem, particularly in the presence of hematoma or when the clinical examination indicates a functional disorder without evidence of structural damage. ${ }^{16}$

In this study, USE showed areas of edema in nine lesions, which were negative at the US examination and therefore classified as FIMD. Comparing these data with the recovery time results, it can be highlighted that the players affected by these nine injuries took more time to return to physical activity compared with others with injuries classified into the same group.

We can therefore distinguish two subgroups within the FIMD group: one subgroup with both US and USE negative, and another subgroup with negative US but positive USE (presence of red colored areas), with the presence of edema areas not assessable with US. This type of injury showed longer time of healing than the first type (-Fig. 5). We may suppose that these injuries represent pre-3a-type injuries because of the absence of confirmed structural injuries at US examination, as required by the Mueller-Wohlfahrt et al's classification. ${ }^{8}$ In this study, $50 \%$ of the lesions classified as 1 a exhibited these characteristics. These lesions were included in a group of "preinjury," before $3 a$ and within the group of structural lesions, and they could be the prelude to a lesion because the presence of edema and soft tissue represent an inflammatory condition and probably an existing injury.

Our interpretation of this kind of injuries might be relevant for the treatment program and for the outcome. Therefore, USE can be a valuable aid in the diagnosis and prognostic evaluation of muscle injuries because it clarifies the staging of the lesion, defining what is not perceptible with the simple B-mode US exam. USE allows for a better definition of the acute phase and the degree of injury, particularly in 1a lesions. Through this additional clinical information, it allows to improve the diagnosis and helps the medical staff to plan a better functional recovery, targeted to the type of lesion. Nonetheless, we conceive that the potential benefit of USE in the better definition of muscle injuries should be deeply investigated in further studies before using it routinely in sports traumatology.

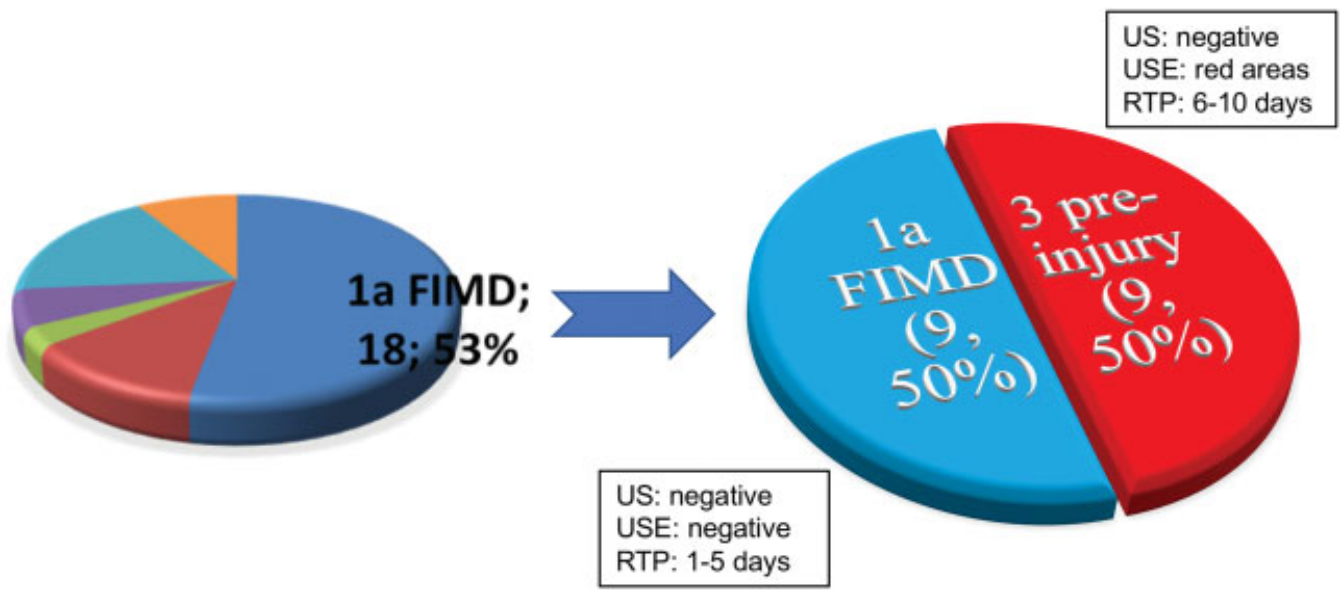

Fig. 5 Fifty per cent of the lesions classified as 1a exhibited characteristics of " pretype-3 injuries" in terms of USE (red area) and of time loss and return to play. FIMD, fatigue-induced muscle disorder; US, ultrasound; USE, elastosonography. 


\section{Conflict of Interest}

None.

\section{References}

1 Menetrey J, Kasemkijwattana C, Day CS, et al. Growth factors improve muscle healing in vivo. J Bone Joint Surg Br 2000;82(01): 131-137

2 Järvinen TA, Järvinen TL, Kääriäinen $M$, et al. Muscle injuries: optimising recovery. Best Pract Res Clin Rheumatol 2007;21(02): 317-331

3 Järvinen TA, Järvinen TL, Kääriäinen M, Kalimo H, Järvinen M. Muscle injuries: biology and treatment. Am J Sports Med 2005;33 (05):745-764

4 Dubowitz V. Muscle Biopsy: A Practical Approach, 2nd ed. Cap 5 129-181. London, Toronto: W. B. Saunders; 1985

5 Gigante A, Cianforlini M, Manzotti S, Ulisse S. The effects of growth factors on skeletal muscle lesions. Joints 2014;1(04): 180-186

6 Järvinen TA, Järvinen TL, Kääriäinen M, et al. Muscle injuries: optimising recovery. Best Pract Res Clin Rheumatol 2007;21(02): 317-331

7 Niitsu M, Michizaki A, Endo A, Takei H, Yanagisawa O. Muscle hardness measurement by using ultrasound elastography: a feasibility study. Acta Radiol 2011;52(01):99-105
8 Mueller-Wohlfahrt HW, Haensel L, Mithoefer K, et al. Terminology and classification of muscle injuries in sport: the Munich consensus statement. Br J Sports Med 2013;47(06):342-350

9 Maffulli N, Del Buono A, Oliva F, et al. Muscle injuries: a brief guide to classification and management. Transl Med UniSa 2014;12:14-18

10 Ekstrand J, Healy JC, Waldén M, Lee JC, English B, Hägglund M. Hamstring muscle injuries in professional football: the correlation of MRI findings with return to play. Br J Sports Med 2012;46 (02):112-117

11 Kuyala UM, Orava S, Jarvinen M. Hamstring injuries. Current trends in treatment and prevention. Sport Med 1997;23(06): 397-404

12 Cianforlini M, Mattioli-Belmonte M, Manzotti S, et al. Effect of platelet rich plasma concentration on skeletal muscle regeneration: an experimental study. J Biol Regul Homeost Agents 2015;29 (4, Suppl):47-55

13 Peetrons P. Ultrasound of muscles. Eur Radiol 2002;12(01):35-43

14 Ekstrand J, Askling C, Magnusson H, Mithoefer K. Return to play after thigh muscle injury in elite football players: implementation and validation of the Munich muscle injury classification. $\mathrm{Br} \mathrm{J}$ Sports Med 2013;47(12):769-774

15 Monetti G, Rampino G, Rusignuolo G. Ultrasound of muscle and tendon pathology. In: Canata GL, d'Hooghe P, Hunt KJ, eds. Muscle and Tendon Injuries. Berlin, Heidelberg: Springer; 2017:75-86

16 Kneeland JP. MR imaging of muscle and tendon injury. Eur J Radiol 1997;25(03):198-208 